\title{
6.3 CAN COMETS BE THE ONLY SOURCE OF INTERPLANETARY DUST?
}

\author{
A.H. Delsemme \\ Department of Physics and Astronomy \\ The University of Toledo \\ Toledo, Ohio/USA
}

\begin{abstract}
The steady-state of the meteoritic complex can be achieved by the present input rate of cometary dust, if the long-period and "new" comets release a sizeable fraction of their dust, in grains larger than 100 microns.
\end{abstract}

I want to limit my concluding remarks to the matter of the balance of the budget needed to maintain the meteoritic dust cloud to its present steady state. This is an important topic, since we cannot really guarantee that we understand anything about the origin or the fate of the meteoritic dust cloud, if there are problems balancing its budget. The dissipating mechanisms require a source of 10 tons per second at steady state (Whipple, 1967). Contrarily to the previous belief, the production rate of dust of the short-period comets observed during this century can only explain $2 \%$ of this amount; those observed during the previous century could explain $3 \%$ of $1 t$ (Delsemme, 1976). However, fluctuations from one century to another are large. It makes sense, because short-period comets decay quickly, and their set is replenished by comets captured by Jupiter; it happens that only one comet as bright as comet Halley is likely to be captured every century into a short-period orbit; one comet five magnitudes brighter (as bright as the Great comet of 1577), every 20 millenia, if Vsekhsvyatskil's (1964) statistics have any sense. It is clear that wide statistical fluctuations are going to happen, mainly introduced by the brightest and rarest comets to be captured into short-period orb1ts.

In order to explain the present steady state, we can formulate several hypotheses:

\section{FIRST HYPOTHESIS}

A comet flve magnitudes brighter than Comet Halley was captured less than 20,000 years ago into a short-period orbit. Comets decay fast, and we do not have any direct evidence of such a capture. However, the meteorold stream associated with comet Encke suggests that 1 t has decayed for a long time, and could have been such a great comet a few millenia ago: this was Fred Whipple's (1967) idea, and it should not 
be rejected lightly. The probability of capture of such a bright comet is of the order of 0.005 per century, therefore, among the 100-odd short period comets that we know, the probability that at least one of them represents the remnants of a very bright comet is 0.5 ; this probability is large enough to feel comfortable with an identification with Comet Encke.

\section{SECOND HYPOTHESIS}

The set of long-period and "new" comets produces enough mass in particles large enough not to be ejected out of the solar system by the radiation pressure of the sun. I have shown previously (Delsemme, 1976) that the long-period comets produce an average of 20 tons of dust per second, if the cutoff of their brightness distribution is set at the (observed) absolute magnitude of -2 . If the major portion of this mass were in particles of the millimeter size, so that the light pressure could be neglected, then symmetry arguments on the vector addition of the particle velocity to the comet's velocity show that half of this mass would be ejected on hyperbolic orbits, but 10 tons/ sec would be kept on more or less elongated orbits. These orbits would then decay into the inner solar system, much as the larger bodies' orbits do.

There are possible variations of this hypothesis. For instance:

a) the cutoff in the brightness distribution of comets can be put elsewhere than at those comets that have been historically observed, namely at magnitude -2. If Vsekhsvyatskii's distribution is extrapolated up to magnitude $-7,99 \%$ of the dust can be lost on hyperbolic orbits, and 10 tons/sec still are steadily captured by the inner solar system.

b) Large particles may represent a much larger fraction of the cometary dust than expected before. Of course, we cannot easily detect them in cometary tails because they do not reflect enough light per unit mass, but several circumstantial arguments point to their existence. As a matter of fact, one of the concluding remarks I would like to submit, is that large particles have become fashionable during this colloquium: we must use them to explain some of the cometary antitails; some of the meteor spectra; or the peculiar polarization of the zodiacal light (specular reflection on rather large crystal facets); we even collect them in the upper atmosphere. They obviously are also in the meteor streams even if they do not reflect enough light to be identified in space: we have learned here that $50 \%$ of the 
mass of the new meteor streams lies in particles of the order of a gram.

c) All comets (short period, long period and "new") with perihelia up to Jupiter and probably Saturn, should possibly be included in the balance of the budget. The argument is that, if they cannot vaporize water, they could vaporize huge amounts of other gases more volatile than water. Some other faraway comets have been known to have comas or tails at distances where water could not vaporize. An excellent example is $\mathrm{P} / \mathrm{Schwassmann-Wachmann} \mathrm{I}$. The total population of comets vary in approximate proportion to their perihelion distances; therefore, we multiply it by five if the radius of the vaporization sphere goes from 2 to $10 \mathrm{AU}$. For long-period or "new" comets we would therefore be allowed to loose $90 \%$ of the dust on hyperbolic orbits, and still be left with the desired rate of capture of 10 tons/sec in the inner solar system. As far as short-period comets are concerned, Comet Schwassmann-Wachmann I has the advantage of being a giant comet on a quasi-circular orbit and could explain most of the dust needed if its outbursts represent a steady state that has lasted ten millenia.

d) The hypothesis of large particles has reminded us that we may be wrong to try to balance the budget with what is easily seen; we see the fine component only because it reflects much light. The total mass of the short-period comet set is not known even within two orders of magnitude. However, it is likely to be very much larger than the dust fraction that is dragged away in the cometary tails. If we accept that most of these comets are not going to die into big asteroids, but that a major mass fraction is going to decay into invisible large chunks of smaller and smaller size, as possibly evidenced by the meteoroids associated with Comet Encke; then we certainly may have enough mass to balance our budget. The spectacular phenomenon of the cometary dust tail, this "bagful of nothing" may have distracted our attention from something more important going on: the steady escape of the major mass into unobservable chunks that decay by steps into meteoroid streams.

\section{SUMMARY}

It is clear that the present production rate of dust by the short-period comets cannot provide more than 2 or $3 \%$ of the mass needed to explain the steady state. In particular, my analysis has been independently confirmed by $S$. Röser (1976) during this colloquium.

Therefore, elther a very bright comet was captured by Jupiter some flve or ten thousand years ago. Comet Encke could be its remnants, and the 
zodiacal light is slowly decaying, waiting for the next bright comet to be captured;

or the major fraction of the dust of the short-period comets is not dragged away by the vaporization of water, but by a stuff more volatile than water, like methane or carbon monoxide or dioxide, in a much larger sphere around the sun. Giant Comet P/Schwassmann-Wachmann would then be a good candidate among the short-period comets of large perihelion distance, although its rare outbursts do not suggest a vaporization steady-state;

or the long-period comets contribute a large fraction of the dust captured. They indeed produce a total dust mass which is at least twice what we need; possibly twenty times if we include comets beyond Jupiter; possibly two hundred times if we include unobserved but historically predictable very bright comets. The real question becomes: what is the fraction of their dust lost on hyperbolic orbits? It could be $50 \%$ only if the particles are in the centimeter size range; $90 \%$ in the millimeter size, $99 \%$ in the 100 micron size, so that the contribution of the long-period comets cannot be neglected if the particles dragged away are large, which is becoming an acceptable idea. NSF Grant GP 39259 is gratefully acknowledged.

\section{REFERENCES}

Delsemme, A.H. (1976) IAU Colloquium No. 31, this volume.

Röser, S. (1976) IAU Colloquium No. 31, this volume.

Vsekhsvyatskii, S.K. (1964) Physical Characteristics of Comets, Translated from Russian, Israel Program of Scientific Translation, NASA-TT-F-80.

Whipple, F. (1967) p. 409 in "The Zodiacal Iight and the Interplanetary Medium", edit. J.L. Weinberg, NASA-SP-150. 